\title{
Aeroacoustic Simulations of Tandem Cylinders with Subcritical Spacing
}

\author{
David P. Lockard, Meelan M. Choudhari ${ }^{\dagger}$ Mehdi R. Khorrami ${ }^{\dagger}$ \\ Dan H. Neuhart $\ddagger$ Florence V. Hutcheson§ Thomas F. Brooks $₫$ \\ NASA Langley Research Center, Hampton, VA 23681 \\ and \\ Daniel J. Stead" \\ Lockheed Martin Engineering and Sciences, Hampton, VA 23681
}

\begin{abstract}
Tandem cylinders are being studied because they model a variety of component level interactions of landing gear. The present effort is directed at the case of two identical cylinders with their centroids separated in the streamwise direction by 1.435 diameters. Experiments in the Basic Aerodynamic Research Tunnel and Quiet Flow Facility at NASA Langley Research Center have provided an extensive experimental database of the nearfield flow and radiated noise. The measurements were conducted at a Mach number of 0.1285 and Reynolds number of $1.66 \times 10^{5}$ based on the cylinder diameter. A trip was used on the upstream cylinder to insure a fully turbulent flow separation and, hence, to simulate a major aspect of high Reynolds number flow. The parallel computational effort uses the three-dimensional Navier-Stokes solver CFL3D with a hybrid, zonal turbulence model that turns off the turbulence production term everywhere except in a narrow ring surrounding solid surfaces. The experiments exhibited an asymmetry in the surface pressure that was persistent despite attempts to eliminate it through small changes in the configuration. To model the asymmetry, the simulations were run with the cylinder configuration at a nonzero but small angle of attack. The computed results and experiments are in general agreement that vortex shedding for the spacing studied herein is weak relative to that observed at supercritical spacings. Although the shedding was subdued in the simulations, it was still more prominent than in the experiments. Overall, the simulation comparisons with measured near-field data and the radiated acoustics are reasonable, especially if one is concerned with capturing the trends relative to larger cylinder spacings. However, the flow details of the $\mathbf{1 . 4 3 5}$ diameter spacing have not been captured in full even though very fine grid computations have been performed. Some of the discrepancy may be associated with the simulation's inexact representation of the experimental configuration, but numerical and flow modeling errors are also likely contributors to the observed differences.
\end{abstract}

Nomenclature

b span

$D \quad$ cylinder diameter

Fxyz fine grid

$M \quad$ Mach number

$M x y z \quad$ medium grid

$M x y F z$ medium grid in $\mathrm{x}-\mathrm{y}$ plane, fine in the span

$p \quad$ pressure

$u, v, w$ Cartesian fluid velocity components

$U, V, W$ time-averaged velocity components

$x, y, z \quad$ Cartesian coordinates
Greek:

$\rho \quad$ fluid density

$\theta \quad$ azimuthal angle

Superscript:

' perturbation quantity (e.g. $\rho^{\prime}=\rho-\rho_{\infty}$ )

Subscript:

$\infty \quad$ freestream quantity

\footnotetext{
${ }^{*}$ Aerospace Technologist, Computational AeroSciences Branch, Mail Stop 128, Senior Member, AIAA

${ }^{\dagger}$ Aerospace Technologist, Computational AeroSciences Branch, Mail Stop 128, Associate Fellow, AIAA

$\ddagger$ Aerospace Technologist, Flow Physics and Controls Branch

$\S$ Aerospace Technologist, Aeroacoustics Branch, Mail Stop 461, Member, AIAA

『Aerospace Technologist, Aeroacoustics Branch, Mail Stop 461, Fellow, AIAA

\| Aerospace Engineer, Aeroacoustics Branch, Mail Stop 461
} 


\section{Introduction}

In an effort to better understand landing gear noise sources, the NASA airframe noise team has been examining a simplified configuration that still maintains some of the salient features of landing gear flow fields. In particular, tandem cylinders have been studied because they model a variety of component level interactions encountered on aircraft undercarriages. The present effort is directed at the case of two identical cylinders spatially separated in the streamwise direction. Extensive experimental data ${ }^{1-4}$ have been collected to investigate the flow fields (especially from the standpoint of noise generation), but also to provide benchmark data to assess the ability of computational simulations to capture the relevant features of such flows. An extensive review of the literature on cylinder flows can be found in Zdravkovich, ${ }^{5,6}$ and work of particular relevance to the current study on tandem cylinders is given in references 7 and 8. A detailed comparison of experimental and computational results for a streamwise spacing of 3.7 diameters was presented in Lockard et al. ${ }^{4}$ In the current investigation of tandem cylinders with their centroids separated in the streamwise direction by 1.435 diameters, we extend our previous work by examining longer span lengths and by making comparisons with aeroacoustic measurements from the QFF. Earlier computational results and their comparison with both flow and acoustic predictions have been presented by Liu et al. ${ }^{9}$ Sheta et al.,${ }^{10}$ Frendi et al. ${ }^{11}$ and Khorrami et al. ${ }^{12}$

The Basic Aerodynamic Research Tunnel (BART) and Quiet Flow Facility (QFF) at NASA Langley Research Center (LaRC) have been used to gather the experimental data. The parallel computational effort by Khorrami et al. ${ }^{13,14}$ initially was restricted to two-dimensional (2-D), fully turbulent simulations using standard, unsteady Reynolds averaged Navier-Stokes (URANS) equations in conjunction with Menter's ${ }^{15}$ two-equation, Shear Stress Transport (SST) turbulence model. Separation distances of $\mathrm{L} / \mathrm{D}=1.435$ and 3.7 were considered, where $\mathrm{D}$ is the diameter and $\mathrm{L}$ the distance between the cylinder centroids. Zdravkovich ${ }^{8}$ characterizes flow patterns associated with various separation distances between the cylinders. For $\mathrm{L} / \mathrm{D}<1.1$, the two cylinders behave as a single bluff body with vortex shedding only occurring behind the rear cylinder. For $1.1<\mathrm{L} / \mathrm{D}<1.6$, the shear layers from the front cylinder attach to the rear cylinder alternately or intermittently, and vortex shedding occurs behind the rear cylinder. For $1.6<\mathrm{L} / \mathrm{D}<2.5$, the shear layers from the front cylinder remain attached to the rear cylinder, and vortex shedding occurs behind the rear cylinder. For separation distances, $2.5<\mathrm{L} / \mathrm{D}<3.2$, intermittent shedding can be detected in the region between the cylinders, and vortex shedding occurs behind the rear cylinder. For $3.2<\mathrm{L} / \mathrm{D}<3.8$, the flow between the cylinders is bistable and switches between intermittent shedding and constant shedding. For L/D $>3.8$, vortex shedding occurs on both cylinders with the same characteristics as a single cylinder. The behavior within these regimes, and the spacings used to define them, can vary as the exact flow behavior is dependent on the Reynolds number, span length, and freestream turbulence. The case of the subcritical spacing of 1.435D where shedding only occurs behind the downstream cylinder is perhaps more relevant in the context of a typical landing gear and forms the subject of this paper.

The 2-D computational results ${ }^{13}$ for 3.7D and 1.435D spacings showed reasonable overall agreement with the measured data but failed to match the details, especially those of the off-surface mean and fluctuating velocity fields. Apart from the shortcomings associated with the 2-D nature of the simulations, those computations produced very strong, coherent shedding without any small scale structures, presumably because of the overly diffusive nature of the turbulence model. Considerable improvement in the comparisons with experiment was achieved by Khorrami et al. ${ }^{12}$ by extending the computations to three-dimensions and using a hybrid, zonal turbulence model that turned off the turbulence production term everywhere except in a narrow ring surrounding the solid surfaces. Due to the high computational cost of the 3D simulations, the spanwise extent of the computational domain was restricted to 3 cylinder diameters. Computations with the hybrid turbulence model more faithfully reproduced the three dimensionality in the flow, in contrast to 3-D URANS calculations that had shown almost no three-dimensional behavior.

\section{Experiments}

The simulated tandem cylinder configuration is comprised of two cylinders of equal diameter (D) aligned along the streamwise direction. The geometry under consideration is shown in figure 1 . The separation distance, L, between the cylinder centroids is $1.435 \mathrm{D}$. The diameter of the cylinders is 2.25 inches $(0.05715 \mathrm{~m})$. A separation distance of 3.7D has been examined previously, ${ }^{4}$ but will not be addressed in this paper. The angle $\theta$ is measured from the upstream stagnation point and increases along the clockwise direction.

Experiments by Jenkins et al. ${ }^{1,2}$ were performed in the BART, providing steady surface pressures, detailed offsurface measurements of the flowfield using Particle Image Velocimetry (PIV), hot-wire measurements in the wake of the downstream cylinder, and unsteady surface pressure data. The BART is a subsonic, atmospheric wind tunnel used to investigate the fundamental characteristics of complex flowfields (see figure 2(a)). The tunnel has a closed test section with a height of 28 inches $(0.711 \mathrm{~m})$, a width of 40 inches $(1.016 \mathrm{~m})$, and a length of 120 inches $(3.048 \mathrm{~m})$. The freestream velocity was set to $144 \mathrm{ft} / \mathrm{s}(44 \mathrm{~m} / \mathrm{s}$, Mach=0.129) to achieve a Reynolds number based on cylinder diameter of 
$1.66 \times 10^{5}$. At these conditions the free stream turbulence level was less than $0.10 \%$. The cylinders spanned the entire BART tunnel height, such that the aspect ratio during the experiment was $\mathrm{b} / \mathrm{D}=12.4$, where $\mathrm{b}$ is the cylinder span. To ensure a fully turbulent shedding process, the boundary layers on the upstream cylinder were tripped between azimuthal locations of 50 and 60 degrees from the leading stagnation point. The measured surface pressure distribution for the large separation case was nearly identical to that measured by previous investigators for a single, isolated cylinder at a Reynolds number greater than 8 million. ${ }^{16}$

Unsteady surface pressure data were obtained from Endevco transducers. The data from both a spanwise row (positioned at $135^{\circ}$ )and $45^{\circ}$ azimuthally spaced ring of sensors were obtained simultaneously. New data from a 2007 entry that focused on more refined azimuthal resolution of the unsteady surface pressure is included in the current paper. The cylinders were rotated in $5^{\circ}$ increments to obtain data at other angles. The rotations provided more refined information about the azimuthal variation of the unsteady pressure from the centerline ring as well as the spanwise row of transducers. Additional information about the BART experiments can be found in References 1,2, and a forthcoming NASA technical report.

Previous QFF measurements documenting the fluctuations in
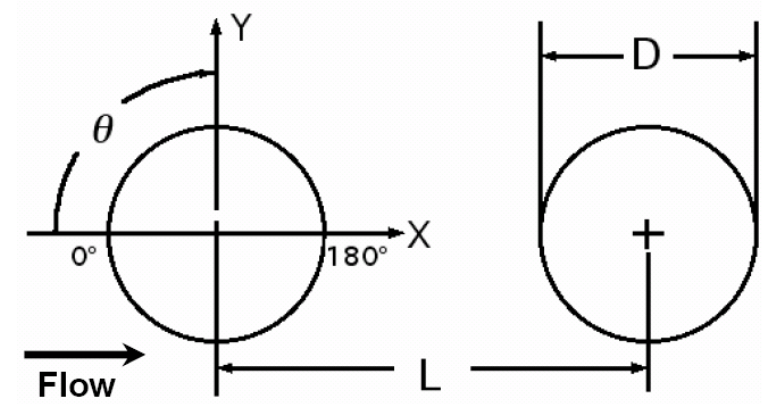

Figure 1. Schematic of tandem cylinder configuration. the wake and the radiated sound field for various isolated cylinder arrangements have been performed by Hutcheson and Brooks. ${ }^{3}$ Results for the tandem cylinder arrangement with a spacing of 3.7D were investigated in the QFF and reported in reference 4. The QFF is an open jet facility specifically designed for anechoic testing and is equipped with a $2 \mathrm{ft}(0.61 \mathrm{~m}) \times 3 \mathrm{ft}(0.914 \mathrm{~m})$ rectangular open jet nozzle. The test models were supported above the nozzle by two vertical side plates that were attached to the short sides of the nozzle (see figure 2(b)). The cylinders spanned the $3 \mathrm{ft}(0.914 \mathrm{~m}$ ) test section yielding an aspect ratio of b/D = 16 (i.e. a 29\% greater aspect ratio compared with the BART configuration). To match the BART results as closely as possible, trips were placed on the front cylinder in the same manner as the BART test, and the speed in the tunnel was adjusted to obtain the surface pressure distribution observed in the BART experiment. The nominal Mach number for the QFF test was $0.1285(142 \mathrm{ft} / \mathrm{s}$ or $43.4 \mathrm{~m} / \mathrm{s})$ with a dynamic pressure of $0.166 \mathrm{psi}(1145 \mathrm{~Pa})$.

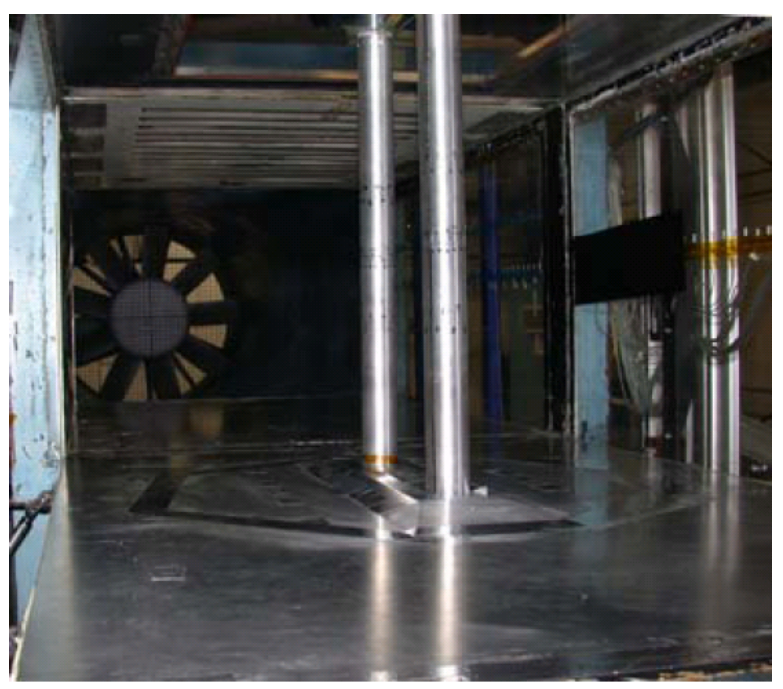

(a) BART

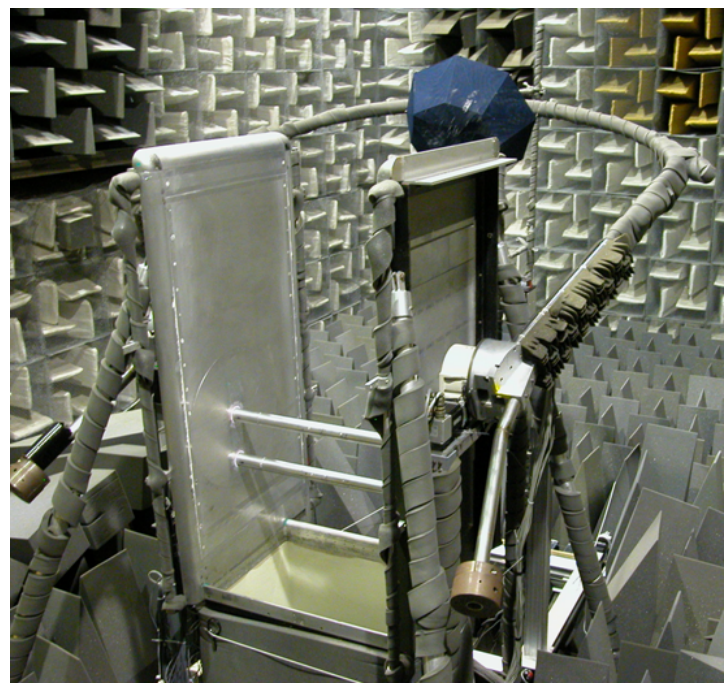

(b) QFF

Figure 2. Tandem cylinder arrangement in the Basic Aerodynamic Research Tunnel and Quiet Flow Facility.

Static and unsteady surface pressure measurements taken in the BART were duplicated in the QFF yielding valuable information about the facility dependence of the results. In addition, six 1/8" microphones mounted on poles near the center of the span of the model were used to collect acoustic data. These microphones were located outside of the jet flow. A 64-channel, 16 bit transient data recorder controlled by a workstation was used to acquire the data at a sampling rate of $25.6 \mathrm{kHz}$. High pass and low pass filters, set respectively at $5 \mathrm{~Hz}$ and $10 \mathrm{kHz}$, were used to condition the outputs from each microphone and Endevco channel. The noise spectra were obtained by partitioning each time signal into 1000 
non-overlapping segments of 8192 samples, and each time history segment was Fourier transformed using a Hamming window for signal conditioning. The resulting frequency resolution was $3.125 \mathrm{~Hz}$.

\section{Computational Simulations}

This study incorporates a coordinated effort between computations and experiment. The time-dependent CFD simulations have been performed using the second-order code CFL3D. ${ }^{17,18}$ CFL3D was developed at NASA LaRC to solve the 3-D, time-dependent, thin layer Reynolds-averaged Navier-Stokes (RANS) equations using a finite-volume formulation. The unsteady calculations use the shear stress transport (SST) $k-\omega$ turbulence model of Menter, ${ }^{15,19}$ which was developed for steady flow. Due to the overly diffusive nature of the turbulence model, results from three-dimensional URANS simulations remained essentially two-dimensional. To remedy this shortcoming, the quasi-laminar approach described in Khorrami et al. ${ }^{12}$ has been adopted for all three-dimensional computations. Following this approach, the flowfield was assumed to be quasi-laminar, except for a narrow strip surrounding the cylinder surfaces where the SST turbulence model was used. Outside of this strip, the production term associated with the turbulence model was switched off. For computational convenience, the distance from the cylinder at which the switch occurs was set based on a set number of grid points from the surface. Because of the azimuthal nonuniformity in the grid stretching, the switch occurs between 0.01D and 0.04D, which includes the entire boundary layer within the region of attached flow. For different grid spacings, the number of points from the surface was changed to maintain the switching at the same physical distance.

The free stream Mach number in the computations was 0.166 , and the Reynolds number was $1.66 \times 10^{5}$ based on the cylinder diameter. The Mach number in the simulations corresponds to the speed used in the first BART tandem cylinder experiments. ${ }^{1}$ Subsequent experiments employed a cylinder of larger diameter to accommodate surface pressure transducers. To maintain the Reynolds number, the experiments were run at slightly different speeds. Therefore, the simulation results have been corrected to an equivalent speed. The BART Mach number of 0.1285 was used as the baseline. Although the experiments used a trip strip to insure turbulent shedding, directly computing the effects of the trip is infeasible. Instead, the computations were run in a fully turbulent mode and allowed to transition on their own. In our calculations, the transition occurs early enough that the flow remains attached to the correct angle for turbulent flow. Even on the rear cylinder, the turbulence model is needed to obtain proper boundary layer development and separation.

\section{III.A. Configurations and Grids}

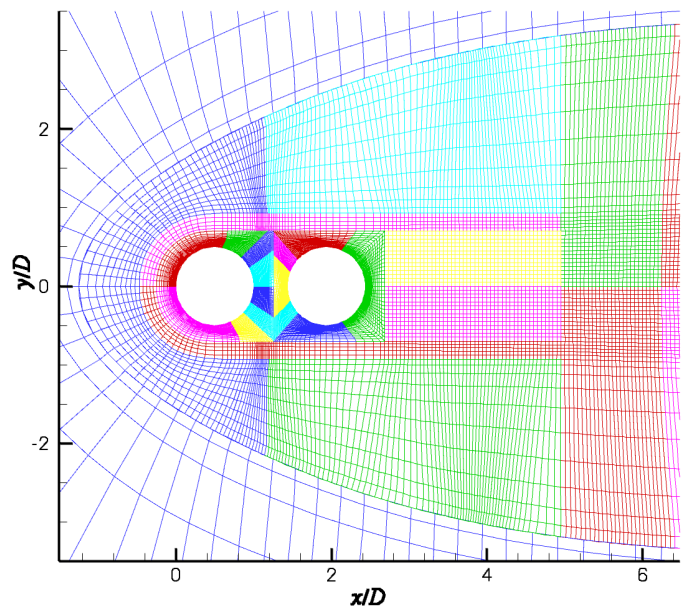

(a) Near field

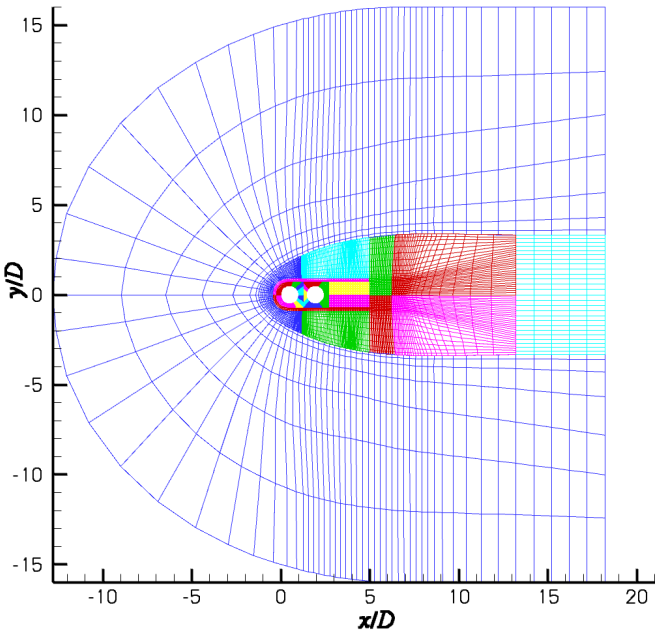

(b) Full grid

Figure 3. Planar view of grid (every 4th point of the Fxyz fine grid).

The tandem cylinders were simulated in free air without any of the wind tunnel walls. The block structured, $x-y$ planar grid shown in figure 3 contained a total of 317,000 points in 29 blocks. Care was taken so that the first point off the solid surfaces was at $y^{+}<1$. To generate the 3-D grids, the planar grids were replicated along the span. The reference length in the grid was the cylinder diameter, D. Hence, a span of 3 means 3 diameters. Periodic boundary conditions were imposed in the spanwise direction. We have not attempted to resolve any of end effects that are present in the experiments. Table 1 summarizes the resolutions and spanwise extents of three families of grids employed in this 
study.

\begin{tabular}{|c|c|c|c|c|c|}
\hline Grid & $x-y$ points & $z$ points & Span & $\Delta z$ & Total points \\
\hline \hline$M x y z$ & $81 \mathrm{E} 3$ & 290 & 18 & 0.0625 & $23.6 \mathrm{E} 6$ \\
\hline$M x y F z$ & $81 \mathrm{E} 3$ & 578 & 18 & 0.03125 & $46.9 \mathrm{E} 6$ \\
\hline Fxyz & $317 \mathrm{E} 3$ & 194 & 6 & 0.03215 & $61.6 \mathrm{E} 6$ \\
\hline
\end{tabular}

Table 1. Resolution and spanwise extent of grids.

The code employs a dual time-stepping algorithm with subiterations used to converge the solution within each time step. We used close to 20 subiterations per time step, varying the number slightly for different grids. CFL3D outputs the subiteration residual, lift, and drag after each subiteration, and we chose the number of subiterations to insure that the lift and drag plateaued within each time step and the residual dropped by 2 to 3 orders of magnitude. However, the convergence of the turbulence quantities was always worse than for the flow equations. A constant non-dimensional time step of $t c_{\infty} / D=0.051$ or $t U_{\infty} / D=0.00847$ was used for most of the simulations. A few of the simulations exhibited strong unsteadiness in the vicinity of the cylinders, and the convergence of the subiteration residual was poor. Therefore, these cases were run at half the time step to achieve a similar drop in the subiteration residual.

The simulation procedure includes several steps. First, a steady-state computation was used to set up the basic mean flow, followed by an unsteady calculation with random suction and blowing applied to different spanwise and azimuthal sections of both cylinder surfaces in order to accelerate the onset of 3D, unsteady flow structures. The forcing did not exceed 3\% of the freestream velocity. The forcing was turned off after shedding was observed, typically 200 time steps into the unsteady calculation. The simulations were then run for 5000-10,000 time steps to allow the transient flowfield to wash out before collecting time records. Averaged flow quantities were produced by time-averaging over 28,00032,000 time steps. The number of averages was increased by taking advantage of the spanwise homogeneity of the flow and spatial-averaging over the span.

\section{Simulating the Experimentally Observed Asymmetry}

Figures 4 (a) and (b) show the experimentally observed surface pressure distributions on the upstream and downstream cylinders, respectively. The QFF and BART pressure distributions on the upstream cylinder agree quite well. However, in comparison with the BART data, the QFF indicates a lower pressure recovery behind the cylinder. The differences in the experimental results may be attributed to the open vs closed wall configurations of the tunnels. Although the experimentally observed $C p$ distributions along the downstream cylinder both display a pronounced asymmetry, the differences between the two sets of data are more noticeable than for the upstream cylinder. In both tests, the asymmetry proved insensitive to small changes in the geometric configuration. To capture the asymmetry, an angle of attack was imposed in the numerical simulations. Figure 4 includes the results from calculations run on the $\mathrm{MxyFz}, \mathrm{span}=18$ grid with angles of attack (AoA) ranging from $0.0^{\circ}$ to $1.5^{\circ}$ in $0.5^{\circ}$ increments. On the upstream cylinder, the asymmetry is slight and all of the calculations show similar agreement with the data. However, the differences between the simulation and experimental results are considerable on the downstream cylinder. Increasing the angle of attack beyond $0.5^{\circ}$ moves the base pressure further away from the experimental result. Nonetheless, the AoA $=1.0-1.5^{\circ}$ cases show the best agreement for the peaks around $270^{\circ}$ and $315^{\circ}$.

Although $C p$ is an important parameter, the purpose of the unsteady simulations is to capture the fluctuating field. The plots of $C p_{r m s}^{\prime}$ Figures 5 (a) and (b) outline the unsteady pressure distribution along the upstream and downstream cylinders, respectively. Because of the significantly higher fluctuation amplitude on the downstream cylinder, the scales are different in the two plots. Again, the two sets of experimental results are in reasonably good agreement with each other. The finer spatial resolution of the BART data resolves several peaks that are not captured in the QFF data. The slight discontinuities in the BART measurements around $\pm 60^{\circ}$ on the upstream cylinder are caused by the trip strips used to insure a fully turbulent boundary layer separation. The calculations were run fully turbulent and were not intended to directly capture the effects of the trips.

Unlike the static pressure results, the rms levels from the simulations at different AoA show considerable variation even on the upstream cylinder. From AoA $=0.0^{\circ}$ to $0.5^{\circ}$, the rms levels change very little, and the agreement with experiment is quite good for both cylinders. However, the predicted unsteady pressure amplitudes for higher AoA are much greater than those observed in both experiments.

Streamlines of the time-averaged flow are presented in figure 6. The BART PIV results in 6 (a) come from reference 2. Although the region downstream of the cylinders is close to symmetric, the streamlines in the gap region between 


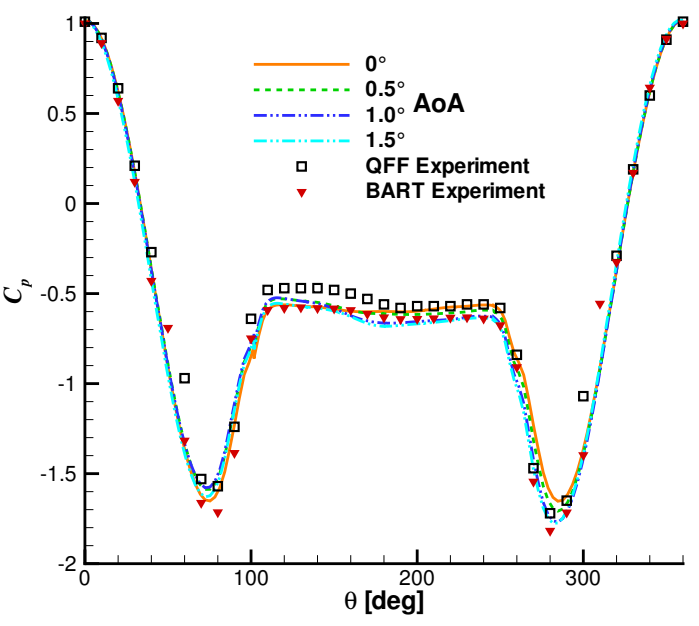

(a) Upstream cylinder

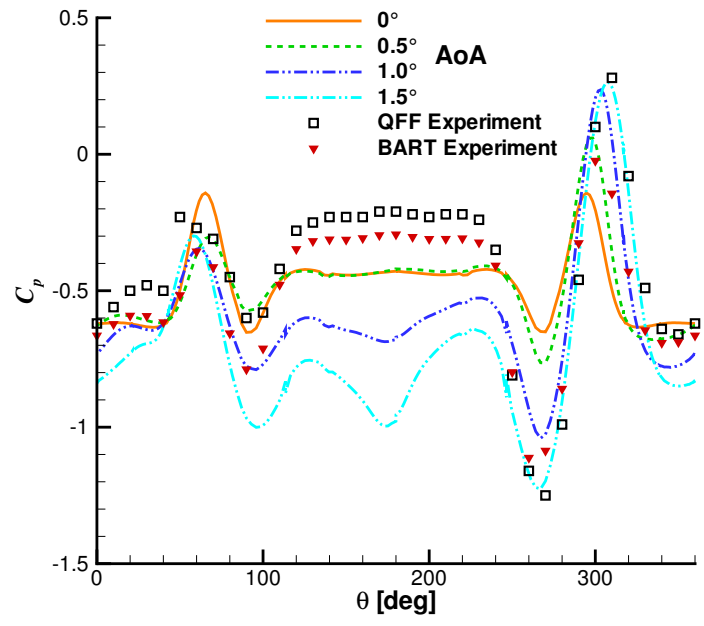

(b) Downstream cylinder

Figure 4. Coefficient of pressure on the surface of the cylinders. Simulation results are from the MxyFz, span=18 grid.

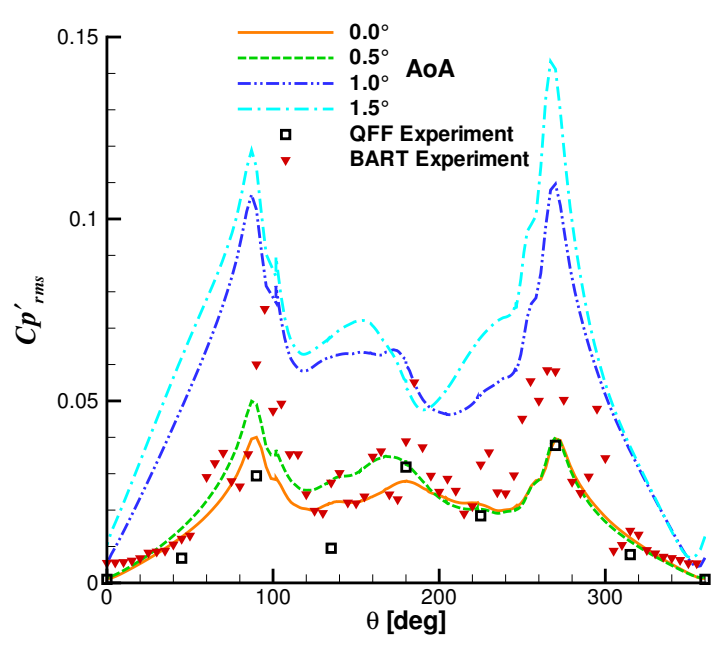

(a) Upstream cylinder

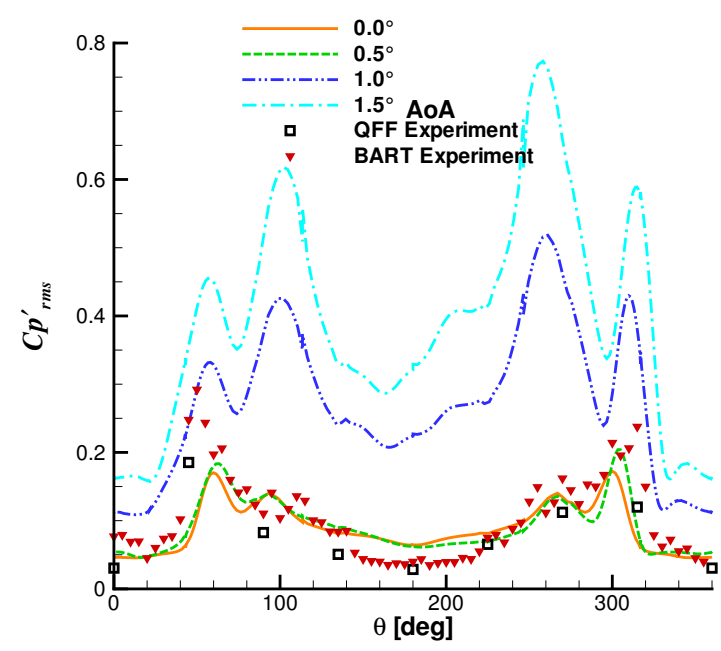

(b) Downstream cylinder

Figure 5. RMS of the coefficient of pressure on the surface of the cylinders. Simulation results are from the MxyFz, span=18 grid.

the cylinders reveals only one primary recirculating zone. Clearly, additional recirculation zones must be present, but they are not captured in the image. The streamline pattern shown in figure $6(b)$ is from the AoA $=0.5^{\circ}$ simulation. At this small AoA, the recirculating region downstream of the cylinders has shortened slightly relative to the symmetric AoA $=0.0^{\circ}$ case (not shown), and the space between the cylinders exhibits 3 recirculating zones resembling the pattern seen in the experiment. However, the position of the primary recirculation is shifted lower and aft of that observed in the experiment. Increasing the angle of attack to $\mathrm{AoA}=1.0^{\circ}$ yields very good agreement between the cylinders, but the downstream wake region is now much shorter than in the experiment. Increasing the AoA to $1.5^{\circ}$ further shrinks the downstream wake.

Based on the static and fluctuating surface pressure results as well as the streamlines, an AoA of $0.5^{\circ}$ appears to be the best compromise to match the observed asymmetry in the experimental data. However, the AoA study was hardly exhaustive, and other configuration changes such as offsetting the cylinders in the vertical direction may produce a similar effect. Furthermore, the effects of spanwise flow may be important for close cylinder spacings, and our 3D simulations with a span of 18 are still not including the effects of the tunnel walls. Each of the calculations with a span 
of 18 was conducted with 46 million grid points and took months to complete on a dedicated cluster with 59 Intel P4 processors. Because of resource constraints, all of the possible causes of the asymmetry and specific flow features could not be investigated. In the remainder of the paper, an AoA of $0.5^{\circ}$ will be used for all the numerical simulations.

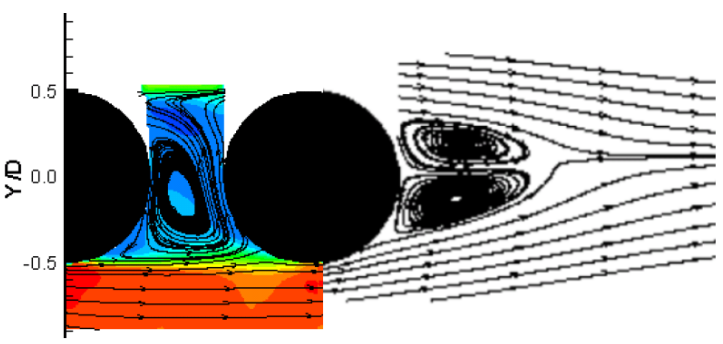

(a) BART Experiment

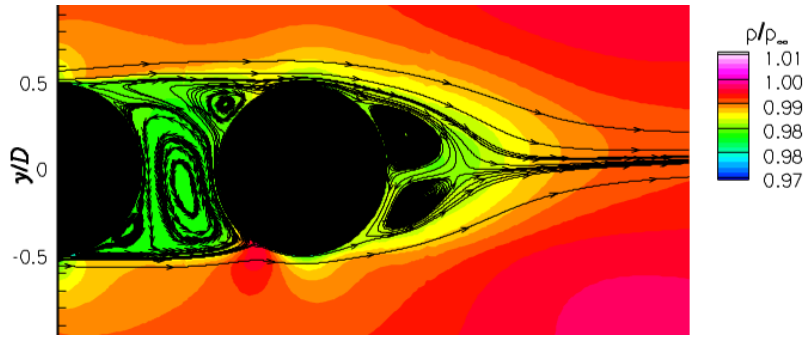

(c) $\mathrm{MxyFz}, \mathrm{Span}=18, \mathrm{AoA}=1.0^{\circ}$

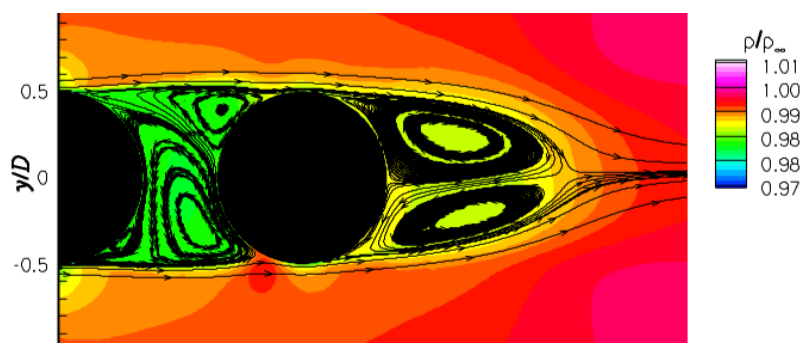

(b) $\mathrm{MxyFz}, \mathrm{Span}=18, \mathrm{AoA}=0.5^{\circ}$

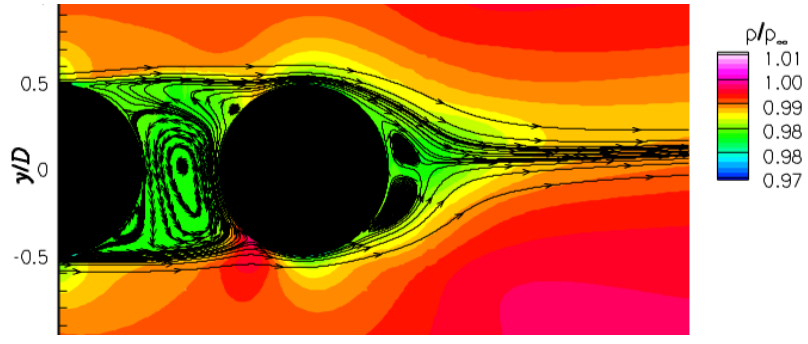

(d) $\mathrm{MxyFz}, \mathrm{Span}=18, \mathrm{AoA}=1.5^{\circ}$

Figure 6. Comparison of computed and experimental streamlines from the time-averaged flow around the cylinders.

\section{IV.A. Surface Pressure}

Figures 7 (a) and (b) present the $C p$ results for the longest spanwise extent used at each of the 3 grid resolutions. The comparison between CFD and experiment on the upstream cylinder is quite good. On the downstream cylinder (figure $7(\mathrm{~b})$ ), the agreement is degraded, but the qualitative aspects of the pressure distributions remain similar. The largest discrepancy is for the Mxyz grid, where the mean base pressure is much lower than for the other two calculations with twice the spanwise resolution.

The $C p_{r m s}^{\prime}$ variations between the different grids are more substantial as seen in figure 8. On the upstream cylinder (figure 8(a)), the fluctuations on the Mxyz grid are higher than those on the other two grids and are well above the experimental data for nearly all azimuthal orientations. Note that the vertical axis range for the downstream cylinder in figure 8(b) is much larger than for the upstream cylinder. On the downstream cylinder, the peak fluctuations in the computations is around $\pm 60^{\circ}$ compared with $\pm 50^{\circ}$ in the BART experiment. This peak is caused by the impingement of the shear layers that bound the region of separated flow between the cylinders. A similar peak in fluctuating surface pressure has been observed in the context of leading-edge slats where the shear layer separating from the cusp reattaches to the slate surface near the trailing edge. ${ }^{20} \mathrm{~A}$ second set of peaks around $\pm 100^{\circ}$ are associated with the boundary layers separating from the surface of the rear cylinder. For the MxyFz solution this peak is small indicating that the shedding off the downstream cylinder is weak. For the Mxyz grid, the rms levels on the entire back side of the downstream cylinder are much higher than for any of the other calculations because it exhibits stronger vortex shedding, and the vortex rollup occurs in close proximity to the cylinder surface.

\section{IV.B. Instantaneous Vorticity}

The time-averaged quantities have revealed the general character of the flow and shown some of the shortcomings of the simulations. Further information about the scales involved in the flow can be ascertained from the instantaneous spanwise vorticity contours in figure 9. The PIV data are dominated by small-scale structures, and large-scale rollup of the shear layers is not evident. A recent test entry in the BART was designed to provide better resolution of the shear layer structures, and should provide useful data for future comparisons. The small scales observed in the BART PIV 


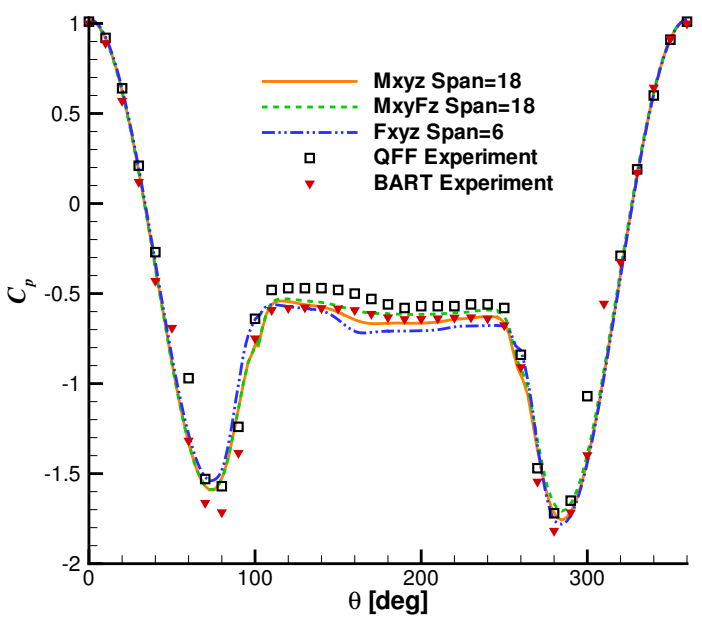

(a) Upstream cylinder

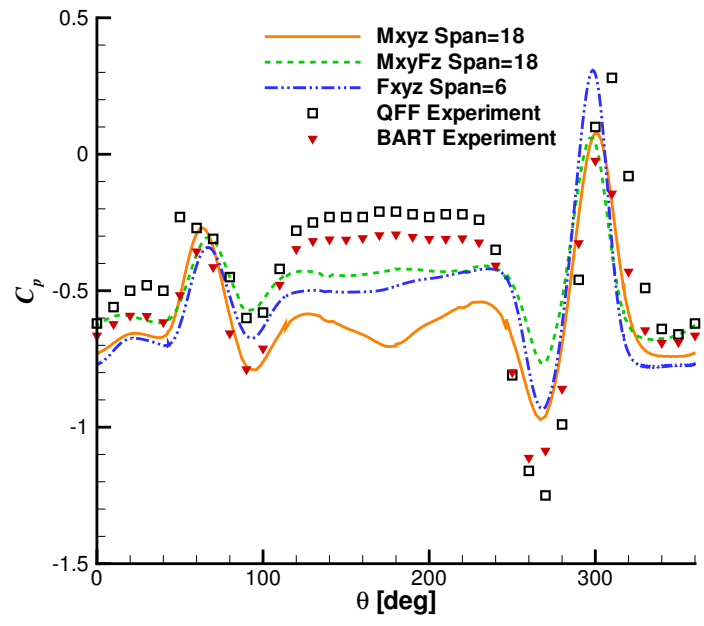

(b) Downstream cylinder

Figure 7. Coefficient of pressure on the surface of the cylinders.

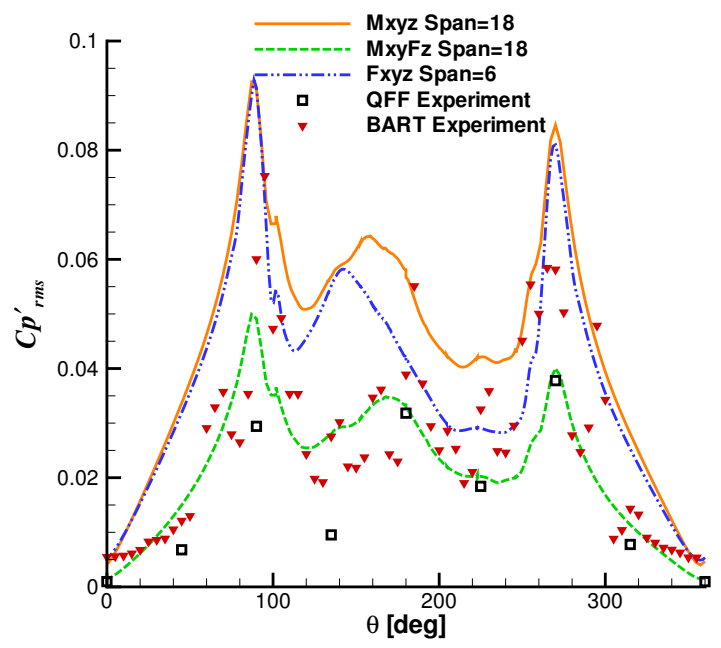

(a) Upstream cylinder

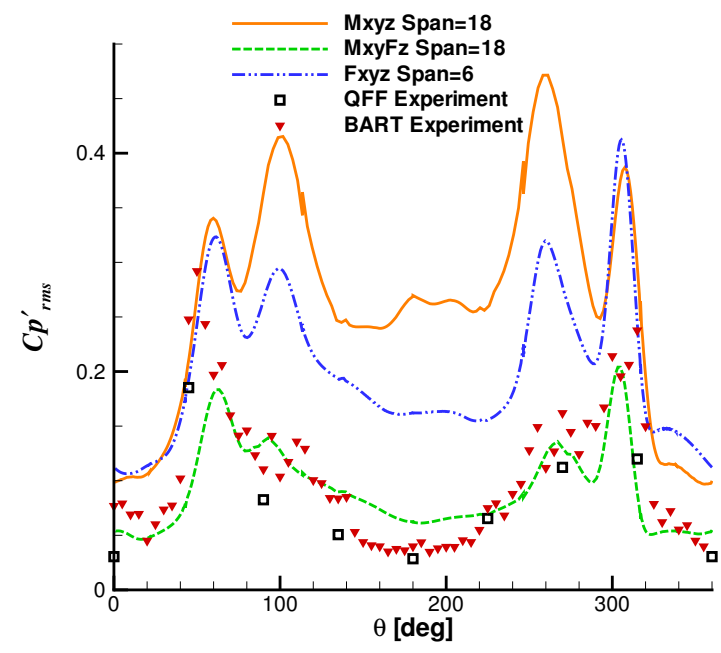

(b) Downstream cylinder

Figure 8. RMS of the coefficient of pressure on the surface of the cylinders.

results are not as common in the simulations, although the finest grid (Fxyz) result does bear some resemblance. In all three simulations, shear-layer rollup is evident in the wake of the rear cylinder. However, animations have revealed that amongst the simulations, the rollup location tends to be much further downstream in the MxyFz case. Also, the unsteadiness in the gap region between the two cylinders is relatively weak and very intermittent on the MxyFz grid. Both of these factors contribute to the relatively low $C p_{r m s}^{\prime}$ values seen in figure 5. Even though the $\mathrm{MxyFz} C p_{r m s}^{\prime}$ values are in good agreement with the experiment, the vorticity contours indicate that the simulation is still missing some finer aspects of the flow.

\section{IV.C. Surface Pressure Spectra}

One can get a much more detailed picture of the surface pressure fluctuations by looking at the spectra. Figures 10 and 11 compare the results from the calculations with the experiments for angles of $45^{\circ}$ and $135^{\circ}$, respectively. The results are presented as power spectral density (PSD). To account for the different freestream velocities in the experiment 


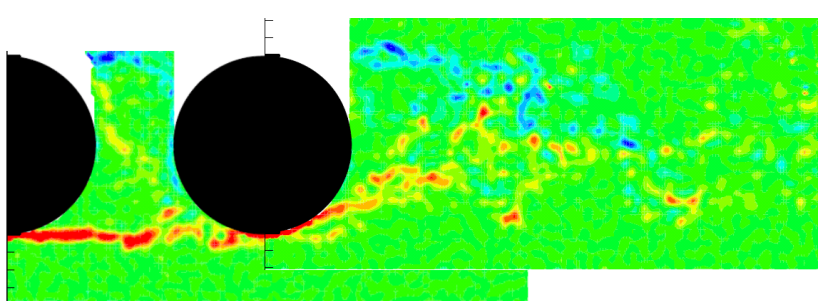

(a) BART Experiment

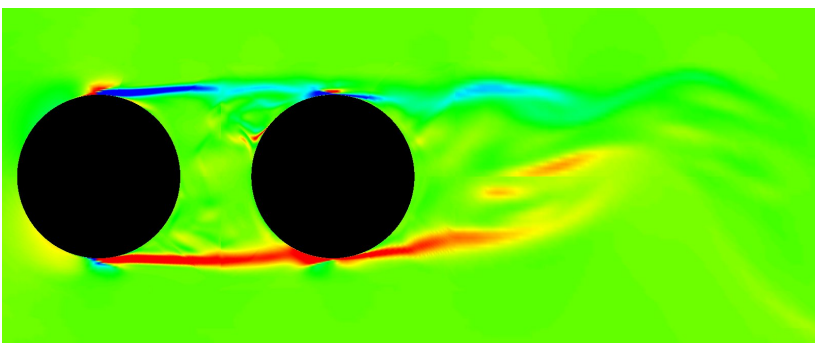

(c) $\mathrm{MxyFz}, \mathrm{Span}=18$

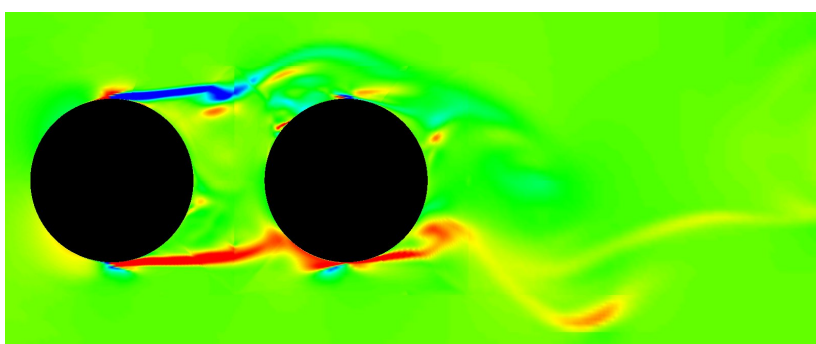

(b) Mxyz, Span=18

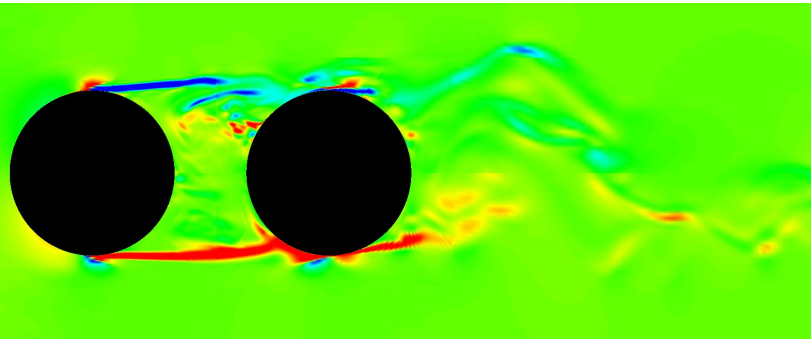

(d) Fxyz, Span=6

Figure 9. Comparison of instantaneous spanwise vorticity contours.

and computation, the CFD pressures were corrected to the experimental condition by assuming that $p \sim|V|^{2}$. Hence, the computed surface pressure was scaled by $(0.1285 / 0.166)^{2}$. The frequency was Strouhal scaled, which results in multiplying the frequency by the velocity ratio. A Hamming filter was applied to each CFD time record, and spanwise averaging was employed. The resulting bin width is $4.15 \mathrm{~Hz}$ ( $3.213 \mathrm{~Hz}$ after scaling), compared with $3.125 \mathrm{~Hz}$ for the experimental data.

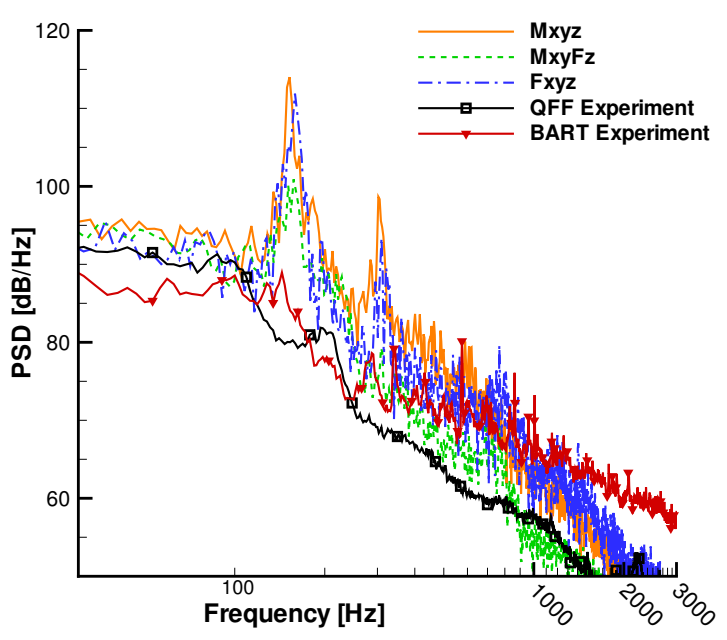

(a) Upstream

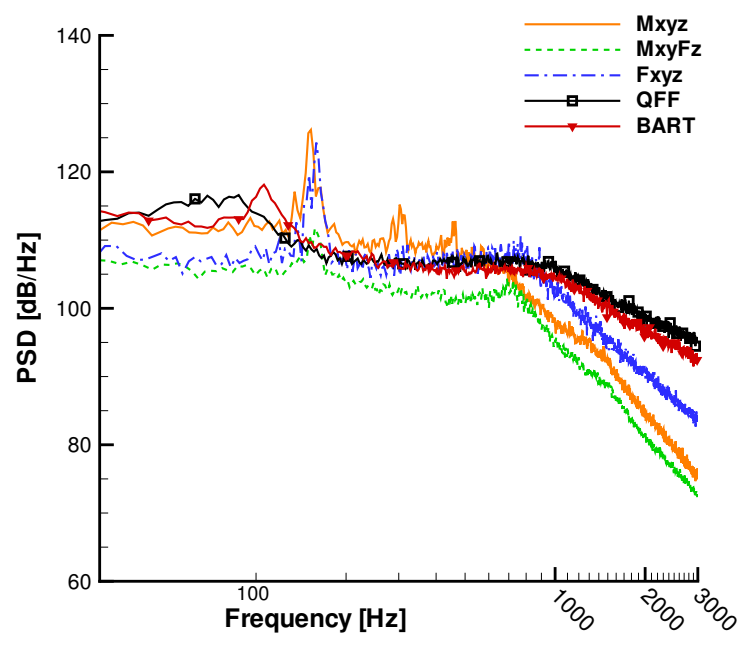

(b) Downstream

Figure 10. Surface pressure spectra on the cylinders at $45^{\circ}$.

At $45^{\circ}$ on the upstream cylinder, the spectral shapes from all 3 simulations are similar, although the exact frequency of the peak around $150 \mathrm{~Hz}$ is slightly different. This peak is caused by vortex shedding in the simulations. The shedding is the strongest on the Mxyz grid, and weakest on the MxyFz grid. The shedding is much weaker in the experiments, although one can still see a small peak around $200 \mathrm{~Hz}$ in the QFF data. We also examined the spectra from the simulations 


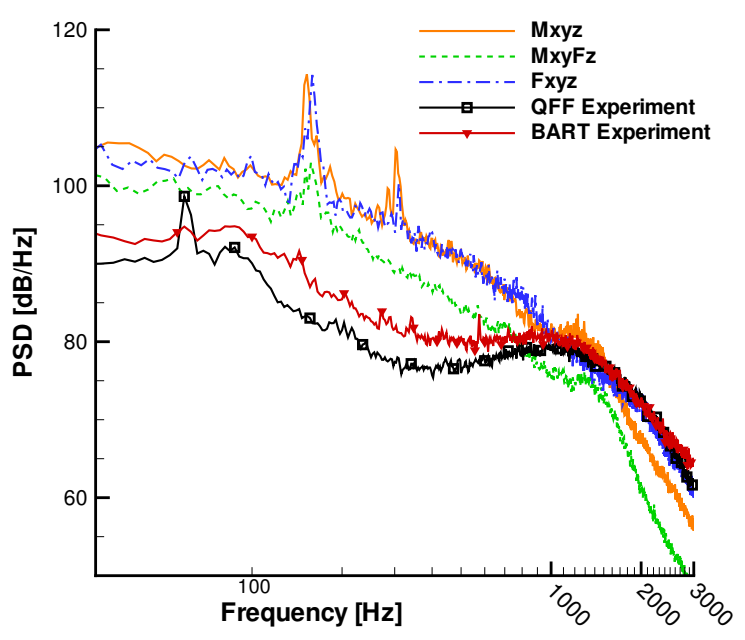

(a) Upstream

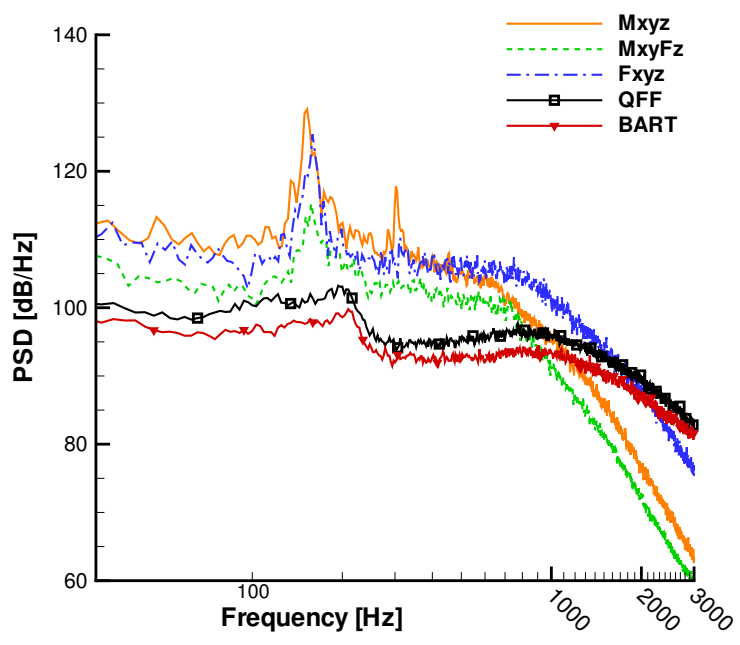

(b) Downstream

Figure 11. Surface pressure spectra on the cylinders at $135^{\circ}$.

at other AoA, and the frequency of the tone is nearly identical between $0.0^{\circ}$ and $0.5^{\circ}$. For higher AoA the tonal frequency shifts to lower frequencies, obtaining a value of $140 \mathrm{~Hz}$ at an AoA of 1.5. Also, the broadband levels increase with increasing AoA. Except for somewhat different amplitudes, the broadband portion of the measured spectra are very similar to the computational spectra. The trip is located between $50-60$, so the flow at this location is still laminar in the experiments. The levels on the downstream cylinder are around $10 \mathrm{~dB}$ higher than on the front cylinder. The shear layer from the upstream cylinder attaches to the downstream cylinder around \pm 45 , and the unsteadiness in the shear layer impacts the cylinder around this point. The experimental spectra from the BART shown in 10 (b) exhibits a small peak around $105 \mathrm{~Hz}$, whereas a broader peak is evident around $90 \mathrm{~Hz}$ in the QFF data. Overall, the experimental spectra are in much better agreement with each other than on the upstream cylinder. The CFD results show a strong peak around $155 \mathrm{~Hz}$, although the amplitude is still smaller than what would be observed for an isolated cylinder. Amongst the CFD results, the peak is smallest for the MxyFz grid. Both the predicted and experimental spectra exhibit a distinctive change in the spectral slope at a frequency around $800 \mathrm{~Hz}$. Although the decay in the CFD spectra at high frequencies may actually be a result of excessive dissipation, some of the effect could be physical.

At $135^{\circ}$ on the upstream cylinder, figure 11 (a) shows the experimental levels agree well and are considerably lower than those from the simulations. Again, the simulations exhibit a tonal character around $155 \mathrm{~Hz}$ that is absent from the experimental data. However, on the downstream cylinder, the experimental spectra do possess a small hump around $195 \mathrm{~Hz}$ and $210 \mathrm{~Hz}$ for the QFF and BART data, respectively. These frequencies are twice those observed at 45. A possible explanation is that shedding from the rear cylinder causes the shear layers between the cylinders to oscillate, and it is the migration of the shear layer across the $45^{\circ}$ location which gives rise to the peak around $100 \mathrm{~Hz}$. In the simulations, animations indicate that this migration occurs irregularly, and it is unclear why it would occur at half the shedding frequency.

\section{IV.D. Correlation}

Information about how well the simulations capture the 3D character of the flowfield in an overall sense can be ascertained from the spanwise correlation of the surface pressure fluctuations,

$$
R_{p p}(\Delta z)=\lim _{T \rightarrow \infty} \int_{0}^{T} p(z, t) p(z+\Delta z, t) d t .
$$

Figure 12 compares the experimental and computational results at $135^{\circ}$ Only the BART experimental results are available for this particular angle. Because periodic boundary conditions are employed in the computations, only half of the span is relevant. Also, the CFD results are averaged over all grid point combinations with the same spanwise spacing. Although the average produces a smooth curve, there is quite a bit of scatter in the CFD data that increases with the separation distance. Similar scatter is also present in the experimental data and has been documented previously. ${ }^{21}$ On 
the upstream cylinder, the CFD correlations drop faster than those in the experiment, but the opposite is true on the downstream cylinder. Only the MxyFz result is able to correctly capture the complete decay in the correlation observed in the experiment. The MxyFz results are well within the scatter observed in the CFD and experiments. The correlation in the Fxyz calculation with a span of only 6D never drops much below 0.4. Even the Mxyz, span=18 calculation does not fully decorrelate to the levels observed in the experiment. Clearly, both sufficient resolution and sufficient spanwise extent are needed in the simulations.

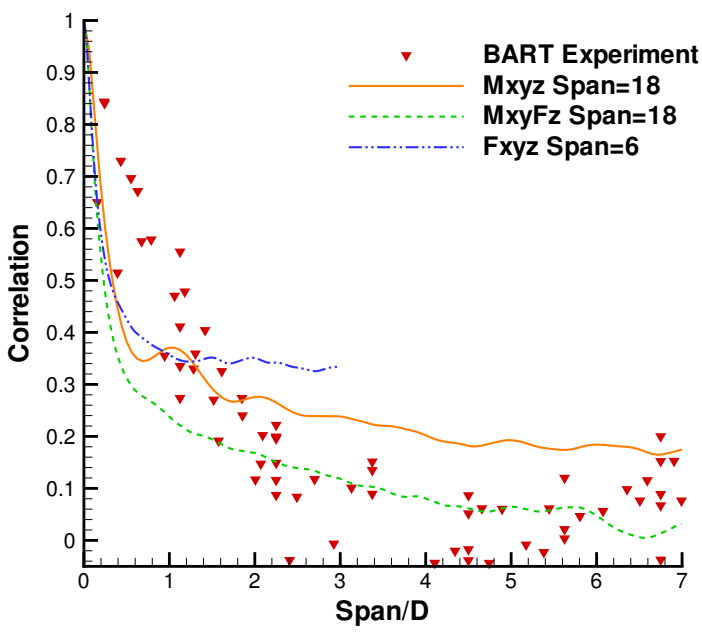

(a) Upstream cylinder

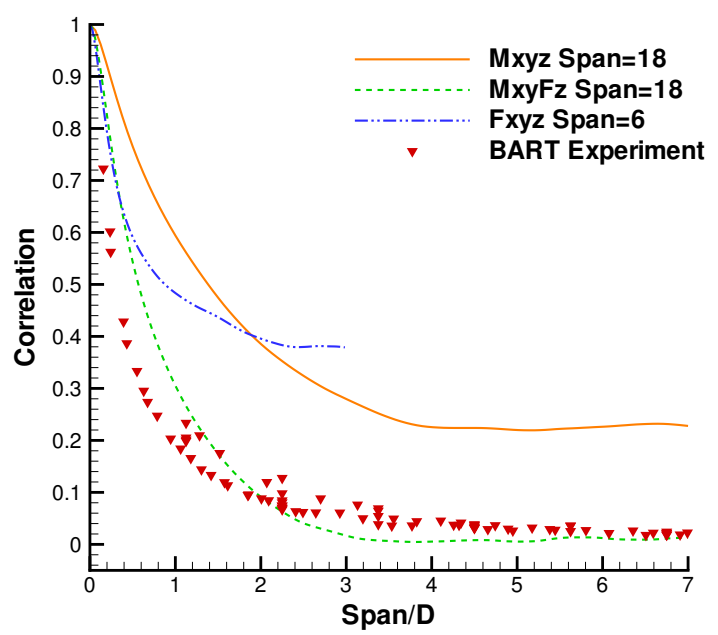

(b) Downstream cylinder

Figure 12. Spanwise correlation comparison at $\theta=135^{\circ}$.

\section{IV.E. Coherence}

Another way to look at the spanwise decorrelation function is through the coherence,

$$
\gamma^{2}(f)=\frac{G_{p_{1} p_{2}}(f)}{\left|G_{p_{1} p_{2}}(f)\right|\left|G_{p_{2} p_{2}}(f)\right|}
$$

where $G p_{1} p_{2}(f)$ is the cross-spectral density function between the pressure at two spanwise locations. The coherence allows one to examine individual frequencies, and there is much less scatter in the data. The coherences at $\theta=135^{\circ}$ for a separation distance of $0.4 \mathrm{D}$ are presented in figure 13. Again, only the BART experimental data is available for this angle. Only the MxyFz simulation results are presented to reduce clutter. The other simulation results are very similar. Also, the coherences at other separation distances are very close in shape, but the amplitudes decrease as the separation distance increases. The experimental coherence on the upstream cylinder is relatively high at very low frequencies and shows a broad peak around $800 \mathrm{~Hz}$. No interesting spectral features can be seen at these frequencies in the spectra shown in figure 11 (a). In contrast, the simulation result shows a distinctive peak around $155 \mathrm{~Hz}$ corresponding to the tone in the surface pressure spectra. The computed coherence near this frequency is similarly high on the downstream cylinder, but the peak is broader. The measured data from the rear cylinder also shows a broad peak in the coherence, but at a higher frequency of $200 \mathrm{~Hz}$. The experimentally observed surface pressure spectra in 11 (b) also exhibit peaks around $200 \mathrm{~Hz}$. Similar coherence and surface pressure spectra have been observed at $225^{\circ}$ giving further evidence of a weak vortex rollup occurring at $200 \mathrm{~Hz}$ in the experiments.

\section{IV.F. Acoustic Radiation}

From a noise perspective, the downstream cylinder will dominate the radiated power because the peak pressure fluctuations are $10 \mathrm{~dB}$ higher than those on the upstream cylinder. The Sound Pressure Level (SPL) contours in the vicinity of the cylinders from figure 14 (a) show that the most intense near-field pressure fluctuations are around $\pm 45^{\circ}$ on the surface of the downstream cylinder and in the wake of the downstream cylinder. The hydrodynamic pressure fluctuations associated with the shear layers bounding the gap region are evident in figure 14 (a). The wake region shows that the fluctuations are more intense in the lower portion of the wake. The asymmetry in the flow gives a strong 


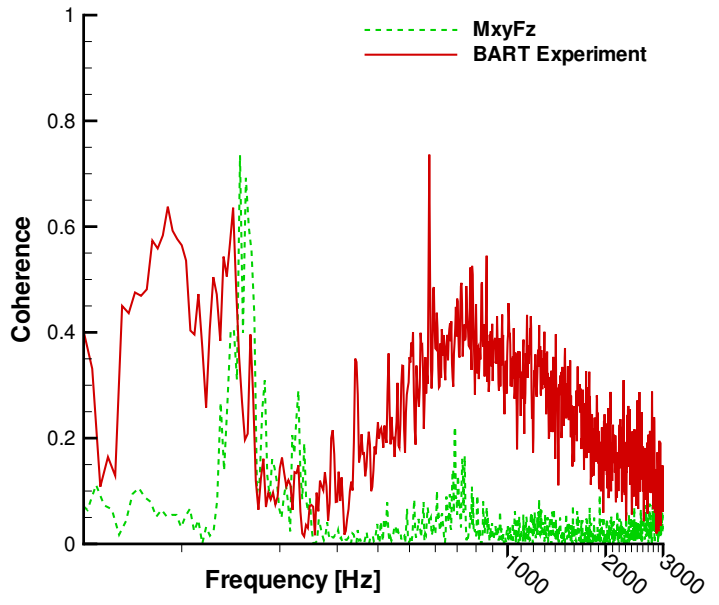

(a) Upstream cylinder

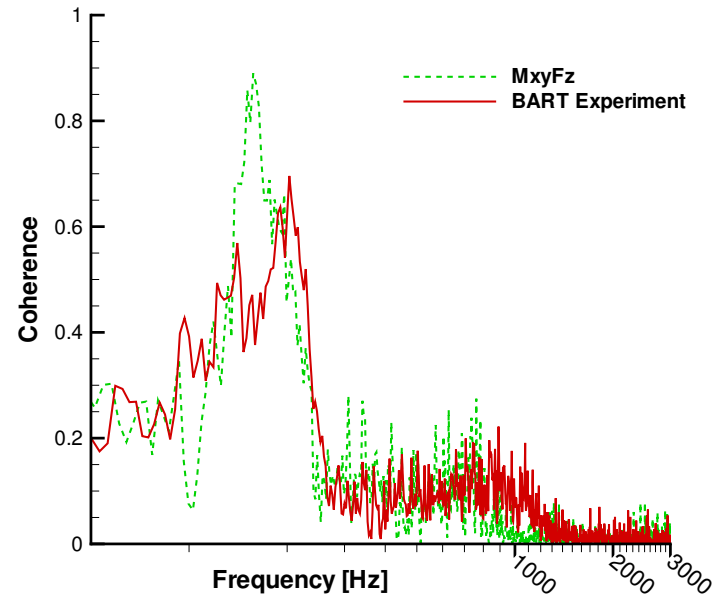

(b) Downstream cylinder

Figure 13. Coherence comparison at $\theta=135^{\circ}$ for a separation distance of $0.4 D$.

preference for the lower shear layer to rollup. Clearly, the CFD has directly captured the radiated noise, but the SPL contours in figure 14 are still very much in the near field. Despite continued advances in computational resources and numerical algorithms, it is still prohibitively expensive and often infeasible to attempt to resolve wave propagation all the way to the observer location. Integral techniques that can predict the far-field signal based solely on near-field input are much more suitable for the prediction of intermediate to far-field acoustics. Hence, the Ffowcs Williams-Hawkings $(\mathrm{FW}-\mathrm{H})$ equation $^{22}$ solver described by Lockard $^{23}$ is used to predict the acoustic signature at various observer locations using the unsteady flow data from the CFD calculations.

The computed directivity pattern using the FW-H equation and solid surface data from the MxyFz, span=18 calculation is presented in figure 14 (b). The CFD predictions are for cylinders in free air and do not include end effects nor the influence of side plates and other mounting hardware used in the experiment. For the directivity plots, the pressure is nondimensionalized by $\rho_{\infty} c_{\infty}^{2}$. The calculations were performed with the frequency-domain version of the FW-H solver using 4096 samples taken over 8192 time steps. To investigate the influence of the statistical convergence of the resulting acoustic predictions, we examined the results from 6 segments of 4096 samples each. For each segment, the source terms to the FW-H equation were formed in the time domain, windowed, and then Fourier transformed. In figure 14 (b), we present the far-field acoustic predictions for each segment separately along with the average of all 6 segments. The $p^{\prime 2}$ results show noticeable differences across different segments, highlighting the intermittency in the time signals. However, on a decibel scale, the differences between the results for any single segment and the average of all the segments fall within $2 \mathrm{~dB}$. Similar variations were investigated in the context of the other grids and found to be somewhat larger than those in figure 14(b) because of the greater fluctuation levels and higher intermittency on those grids.

Table 2 lists the locations of the pole microphones from the QFF experiment. The positions are given relative to the center of the upstream cylinder. The microphones were located along a circular arc, but the centroid was (9.11D, -2.4D), not the geometric center of the model.

Noise predictions from the 3 grids at the 6 pole microphone locations in the QFF experiment are shown in figure 15. The FWH predictions only used the span length from the CFD, so the acoustic amplitude based on the Fxyz grid (which was limited to 6D in the spanwise direction) is somewhat decreased relative to the other, longer span calculations. The frequencies from the CFD spectra have been Strouhal scaled, and the amplitude adjusted assuming a compact dipole scaling of $p^{\prime 2} \sim V^{6}$. The spectra for all the simulations show a distinct peak around $155 \mathrm{~Hz}$ for each of the 6 locations, although there are some differences in the amplitudes of the peaks. The simulation results also show several harmonics of the fundamental, depending on the grid and microphone location. However, the MxyFz spectra has a secondary peak around $220 \mathrm{~Hz}$. There was no evidence of spectral features at this frequency in any of the surface pressure data, so the source of this peak is unknown.

The gradual rise in the experimental spectra below $75 \mathrm{~Hz}$ is most likely a facility effect and should be ignored. The experimental data exhibit peaks around 105 and $205 \mathrm{~Hz}$. The $205 \mathrm{~Hz}$ peak corresponds to the hump in the surface pressure spectra and high coherence at $135^{\circ}$ on the rear cylinder. The $105 \mathrm{~Hz}$ peak may correspond to the higher levels 


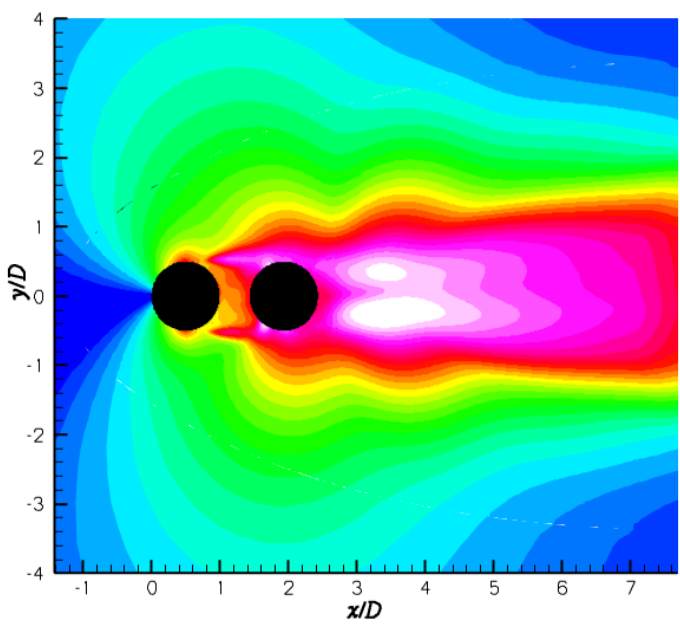

(a) CFD Prediction

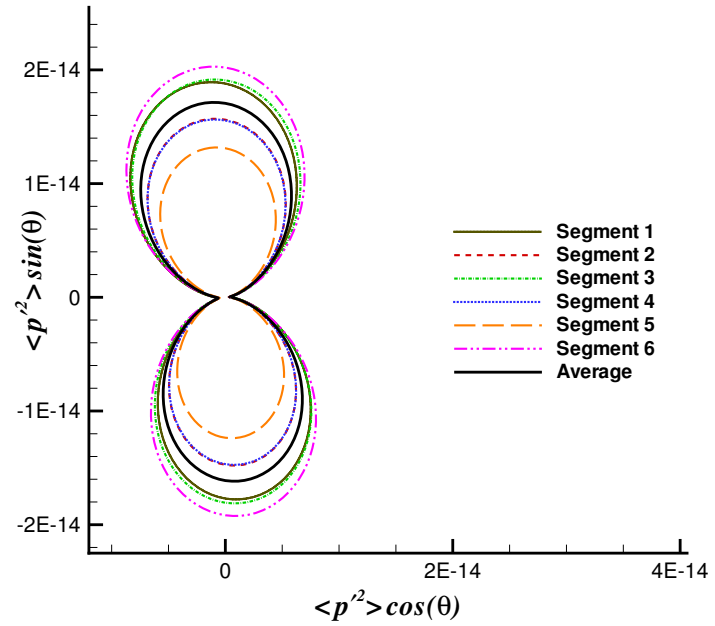

(b) FWH Prediction

Figure 14. Directivity around the cylinders from the MxyFz, span=18 simulation.

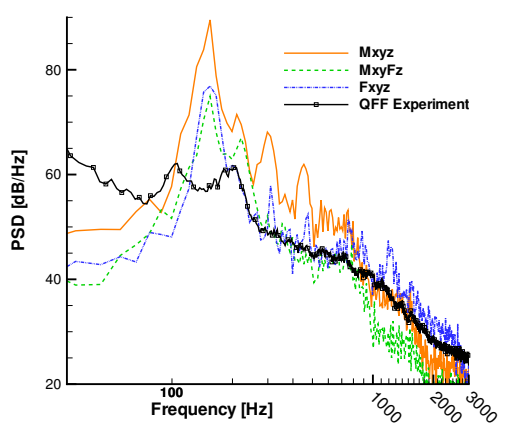

(a) Microphone A

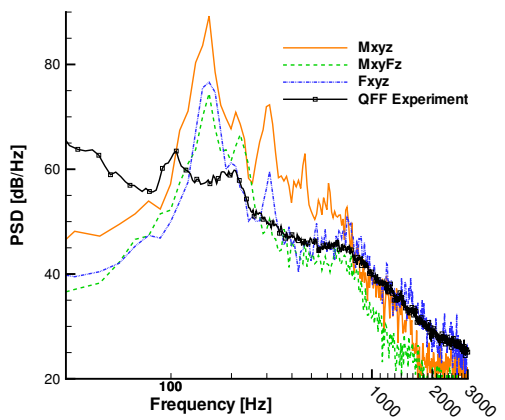

(d) Microphone D

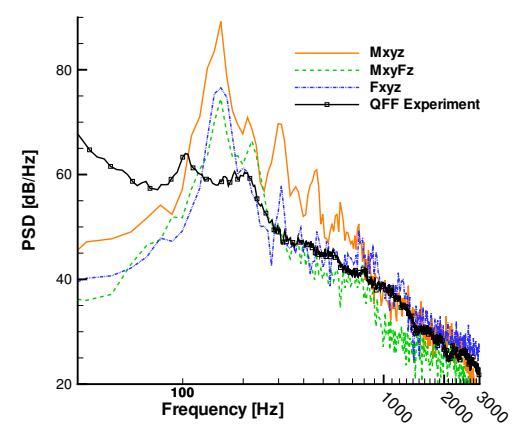

(b) Microphone B

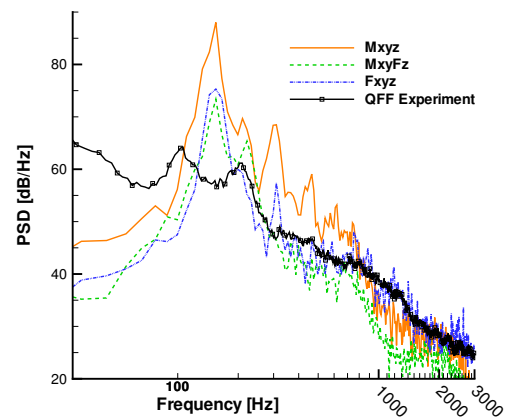

(e) Microphone E

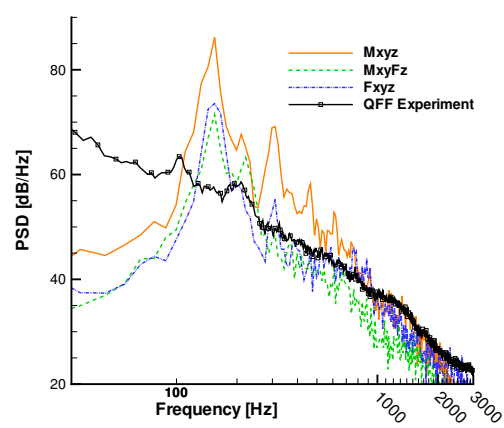

(c) Microphone $\mathrm{C}$

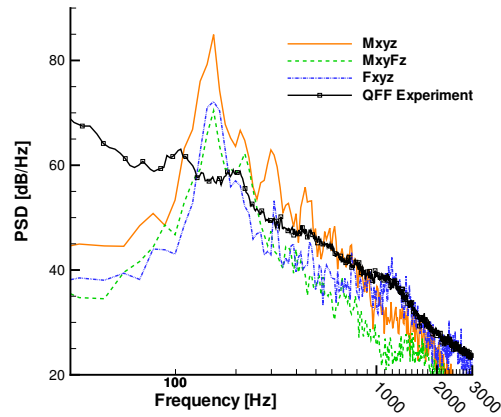

(f) Microphone F

Figure 15. Comparison of computed and experimental spectra at the pole microphone locations. 


\begin{tabular}{|c|c|c|c|c|c|c|}
\hline & $\mathrm{A}$ & $\mathrm{B}$ & $\mathrm{C}$ & $\mathrm{D}$ & $\mathrm{E}$ & $\mathrm{F}$ \\
\hline \hline$x$ & -8.333 & 9.111 & 26.55 & -8.333 & 9.111 & 26.555 \\
\hline$y$ & -27.815 & -32.489 & -27.815 & 32.615 & 37.615 & 32.615 \\
\hline
\end{tabular}

Table 2. Microphone locations from the center of the upstream cylinder. Distances are given relative to the cylinder diameter.

observed at $45^{\circ}$ in the pressure signal on the downstream cylinder, although the frequency is a better match with that observed in the BART than in the QFF.

Although the mismatch in the tonal features between the simulations and experiments is large, the broadband comparison is surprisingly good in terms of spectral shape, especially in the upstream direction. Beyond $800 \mathrm{~Hz}$, the coarser grid solutions decay too rapidly, but the Fxyz result is still quite close to the experiment. The fine grid is presumably capturing more of the fine scales that are responsible for the noise generation at the higher frequencies.

\section{Effect of Cylinder Separation}

The previous discussion has shown that the subcritical spacing of $1.435 \mathrm{D}$ results in a challenging flowfield to simulate all the details. Often, however, the noise engineer is most interested in how the flowfield and radiated noise changes as a result of modifications to the geometric configuration. Thus, we next examine how well the computations predict the changes in salient metrics as the cylinder spacing is varied from supercritical (i.e. $\mathrm{L} / \mathrm{D}=3.7$, which was investigated in reference 4) to subcritical $(\mathrm{L}=1.435)$. We begin by comparing the levels of peak surface pressure on the downstream cylinder in both cases. Using the $\mathrm{MxyFz}, \mathrm{span}=18$ calculation as the reference CFD calculation, a comparison with experimental $C p_{r m s}^{\prime}$ results for the 2 separation distances is shown in figure 16(a). The peak rms levels are different by a factor of 2-3 between the two configurations, and the CFD has picked up this effect. All of the CFD solutions from the different grids correctly capture the trend.

Acoustic results for the different separation distances are compared in figure 16 (b). For both the experimental and CFD results, the tone and broadband amplitudes are significantly lower for the smaller spacing. Decreases of 10-20 on a $\mathrm{dB}$ scale represent orders of magnitude reductions in the fluctuating pressure. Clearly, the CFD has predicted the correct trend in the far-field acoustics even though the details of the spectrum at the smaller spacing have not been captured. Whether the demonstrated capability of the CFD is sufficient is problem dependent. Clearly, not all changes that one would envision for a real landing gear would be as drastic as changing the spacing from 3.7D to 1.435D. Given that $3 \mathrm{~dB}$ is considered important for aircraft design, considerable improvement in the predictive capability of CFD based methods is needed.

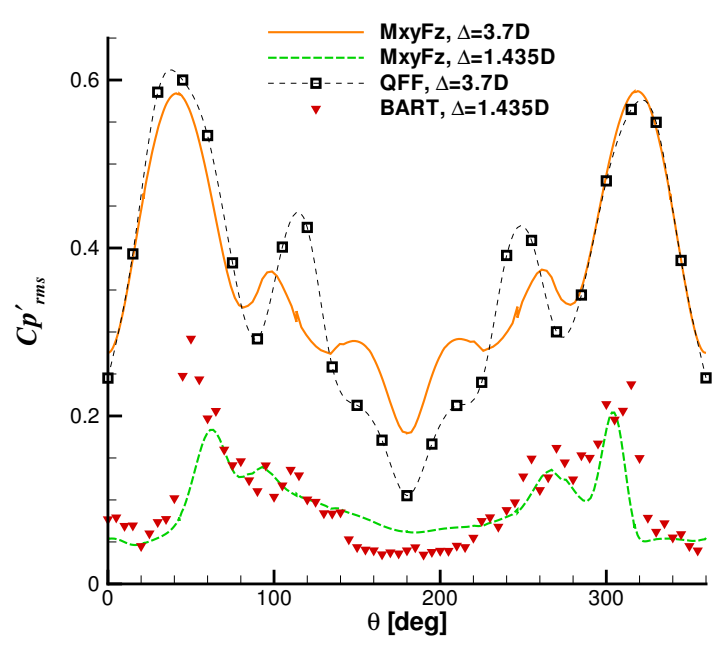

(a) $C p_{r m s}^{\prime}$ on the downstream cylinder

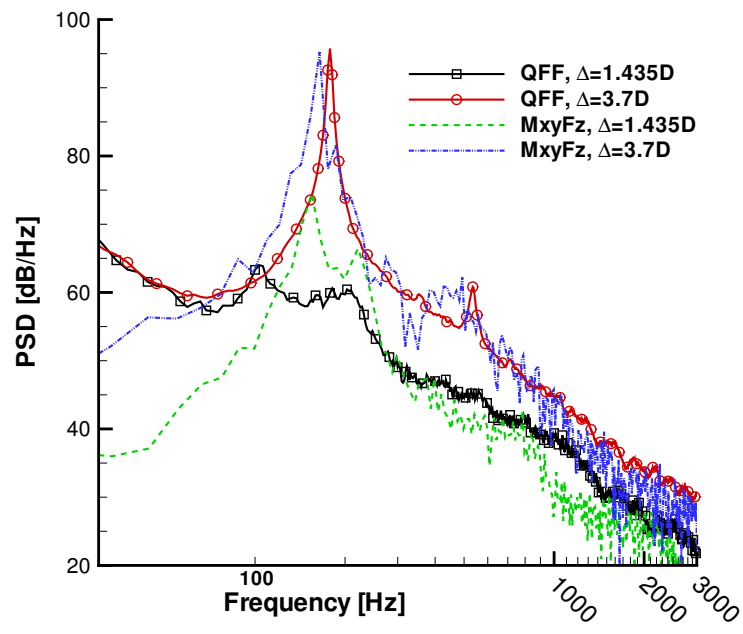

(b)

Figure 16. Comparison of results for 1.435D and 3.7D cylinder spacings. 


\section{Conclusions}

The current simulations have demonstrated the capability of CFD to predict the flow and acoustics around tandem cylinders with a subcritical spacing of 1.435D. Although the experimental results from the BART and QFF are in good agreement, both exhibited an unexpected asymmetry that complicated comparisons with the simulations. The current computations show that reasonable agreement with experiment is possible using an angle of attack of $0.5^{\circ}$; however, further refinement of the configuration parameters and CFD may improve the results.

The 1.435D separation case does not exhibit strong vortex shedding and is likely to be more relevant to real landing gear than configurations with a supercritical spacing. Amongst the 3 simulations on different grids, the MxyFz, span $=18 \mathrm{D}$ results typically compared the best with the measured surface pressure fluctuation levels. However, the vorticity contours from the experiment exhibited finer details than those observed on the MxyFz grid. Only the Fxyz grid was able to partially capture these small-scale, vortical structures. Furthermore, the far-field acoustic results showed that only the fine grid predictions were able to match the shape of the measured spectra above $800 \mathrm{~Hz}$. Because of resource constraints, the fine grid calculation was limited to 6 diameters. Even though the MxyFz result had the best overall agreement with the experiments, several important aspects of the flow were missing, indicating that a fine grid calculation on a longer span may be needed.

Similar to the 3.7D separation case examined earlier, the downstream cylinder sees much larger pressure fluctuations than the upstream cylinder. However, at $1.435 \mathrm{D}$, the shear layer from the upstream cylinder attaches intermittently to the downstream cylinder, and vortex shedding is greatly suppressed. The far-field spectra from the QFF experiment shows two minor peaks around 100 and $200 \mathrm{~Hz}$. The simulations were not able to predict these spectral features and instead show a single, more prominent peak around $155 \mathrm{~Hz}$. Although the simulations were not able to capture the details of the experimental results, they all correctly predicted the observed changes from a supercritical to subcritical spacing. Such a capability is often of value to the designer even if the details of a given flowfield are not completely predicted.

\section{Acknowledgments}

The authors would like to thank Jaye Moen and Dennis Kuchta for their help in acquiring the QFF data, and Donald Day and Luther Jenkins for their help with the BART data.

\section{References}

\footnotetext{
${ }^{1}$ Jenkins, L. N., Khorrami, M. R., Choudhari, M. M., and McGinley, C. B., "Characterization of Unsteady Flow Structures Around Tandem Cylinders for Component Interaction Studies in Airframe Noise," AIAA Paper 2005-2812 Presented at the 11th AIAA/CEAS Aeroacoustics Conference, Monterey, CA, May 23-25, 2005.

${ }^{2}$ Jenkins, L. N., Neuhart, D. H., McGinley, C. B., Choudhari, M. M., and Khorrami, M. R., "Measurements of Unsteady Wake Interference Between Tandem Cylinders," AIAA Paper 2006-3202 Presented at the 36th AIAA Fluid Dynamics Conference and Exhibit, San Francisco, CA, United States, June 5-8, 2006.

${ }^{3}$ Hutcheson, F. V. and Brooks, T. F., "Noise Radiation from Single and Multiple Rod Configurations," AIAA Paper 2006-2629 Presented at the 12th AIAA/CEAS Aeroacoustics Conference, Cambridge, MA, May 8-10, 2006, 2006.

${ }^{4}$ Lockard, D. P., Khorrami, M. R., Choudhari, M. M., Hutcheson, F. V., Brooks, T. F., and Stead, D. J., "Tandem Cylinder Noise Predictions," AIAA Paper 2007-3450, Presented at the 13th AIAA/CEAS Aeroacoustics Conference, Rome, Italy, 2007.

${ }^{5}$ Zdravkovich, M. M., Flow Around Circular Cylinders Volume 1, Oxford University Press, 1997.

${ }^{6}$ Zdravkovich, M. M., Flow Around Circular Cylinders Volume 2: Applications, Oxford University Press, 2002.

${ }^{7}$ Zdravkovich, M. M., "Review of Flow Interference Between Two Circular Cylinders in Various Arrangements," Journal of Fluids Engineering, Vol. 99, 1977, pp. 618-633.

${ }^{8}$ Zdravkovich, M. M., "Flow Induced Oscillations of Two Interfering Circular Cylinders," Journal of Sound and Vibration, Vol. 4, 1985, pp. 511521.

${ }^{9}$ Liu, Q. and Vasilyev, O., "Hybrid Adaptive Wavelet Collocation - Brinkman Penalization Method for Unsteady RANS Simulations of Compressible Flow around Bluff Bodies," AIAA Paper 2006-3206 Presented at the 36th AIAA Fluid Dynamics Conference and Exhibit, San Francisco, CA, United States, June 5-8, 2006.

${ }^{10}$ Sheta, E., Tosh, A., and Habchi, S., "Wavelet-Based Adaptive Multiresolution Methodology for Tandem Cylinder Noise," AIAA Paper 20063205 Presented at the 36th AIAA Fluid Dynamics Conference and Exhibit, San Francisco, CA, United States, June 5-8, 2006.

${ }^{11}$ Frendi, K., Zreik, M., and Tosh, A., “A Computational Study of the Unsteady Flow Past Tandem Cylinders,” AIAA Paper 2006-3204 Presented at the 36th AIAA Fluid Dynamics Conference and Exhibit, San Francisco, CA, United States, June 5-8, 2006.

${ }^{12}$ Khorrami, M. R., Lockard, D. P., Choudhari, M. M., Jenkins, L. N., Neuhart, D. H., and McGinley, C. B., "Simulations Of Bluff Body Flow Interaction For Noise Source Modeling," AIAA Paper 2006-3203 Presented at the 36th AIAA Fluid Dynamics Conference and Exhibit, San Francisco, CA, United States, June 5-8, 2006.

${ }^{13}$ Khorrami, M. R., Choudhari, M. M., Jenkins, L. N., and McGinley, C. B., "Unsteady Flowfield Around Tandem Cylinders as Prototype for Component Interaction in Airframe Noise," AIAA Paper 2005-2866 Presented at the 11th AIAA/CEAS Aeroacoustics Conference, Monterey, CA, May 23-25, 2005.
} 
${ }^{14}$ Khorrami, M. R., Choudhari, M. M., Lockard, D. P., Jenkins, L. N., and McGinley, C. B., "Unsteady Flowfield Around Tandem Cylinders as Prototype for Component Interaction in Airframe Noise," AIAA Journal, Vol. 45, No. 8, 2007, pp. 1930-1941.

${ }^{15}$ Menter, F. R., "Two-equation Eddy-viscosity Turbulence Models for Engineering Applications," AIAA Journal, Vol. 32, No. 8, 1994, pp. 15981605.

${ }^{16}$ Roshko, A., "Experiments On The Flow Past A Circular Cylinder At Very High Reynolds Number," Journal of Fluid Mechanics, Vol. 10, 1961, pp. 345-356.

${ }^{17}$ Rumsey, C. L., Biedron, R. T., and Thomas, J. L., “CFL3D: Its History and Some Recent Applications,” NASA TM 112861, May 1997, presented at the Godonov's Method for Gas Dynamics Symposium, Ann Arbor, MI.

${ }^{18}$ Krist, S. L., Biedron, R. T., and Rumsey, C. L., "CFL3D User's Manual (Version 5)," NASA TM 208444, NASA Langley Research Center: Computational Aerosciences Branch, 1997.

${ }^{19}$ Menter, F. R., "Zonal Two-equation k- $w$ Turbulence Models for Aerodynamic Flows,” AIAA Paper 1993-2906, 1993.

${ }^{20}$ Choudhari, M. M. and Khorrami, M. R., "Effect of Three-Dimensional Shear-Layer Structures on Slat Cove Unsteadiness," AIAA Journal, Vol. 45, No. 9, 2007, pp. 2174-2186.

${ }^{21}$ Szepessy, S., "On the Spanwise Correlation of Vortex Shedding from a Circular Cylinder at High Subcritical Reynolds Number," Physics of Fluids, Vol. 6, No. 7, 1994, pp. 2406-2416.

${ }^{22}$ Ffowcs Williams, J. E. and Hawkings, D. L., "Sound generation by turbulence and surfaces in arbitrary motion," Philosophical Transactions of the Royal Society of London A, Vol. 342, 1969, pp. 264-321.

${ }^{23}$ Lockard, D. P., "A Comparison of Ffowcs Williams-Hawkings Solvers for Airframe Noise Applications," AIAA Paper 2002-2580, 8th AIAA/CEAS Aeroacoustics Conference, Breckenridge, CO, June 17-19, 2002. 